\title{
Multi-gene phylogeny of Madagascar's plated lizards, Zonosaurus and Tracheloptychus (Squamata: Gerrhosauridae)
}

\author{
Hans Recknagel ${ }^{\mathrm{a}, \mathrm{f}}$, Kathryn R. Elmer ${ }^{\mathrm{a}, \mathrm{f}}$, Brice P. Noonan ${ }^{\mathrm{b}}$, Achille P. Raselimanana ${ }^{\mathrm{c}, \mathrm{d}}$, Axel Meyer ${ }^{\mathrm{a}}$, \\ Miguel Vences ${ }^{\mathrm{e}, *}$ \\ ${ }^{a}$ Lehrstuhl für Zoologie und Evolutionsbiologie, Department of Biology, University of Konstanz, 78457 Konstanz, Germany \\ ${ }^{\mathrm{b}}$ Department of Biology, University of Mississippi, Box 1848, University, MS 38677, USA \\ ${ }^{\mathrm{c}}$ Département de Biologie Animale, Université d'Antananarivo, BP 906, Antananarivo (101), Madagascar \\ ${ }^{\mathrm{d}}$ Association Vahatra, BP 3972, Antananarivo (101), Madagascar

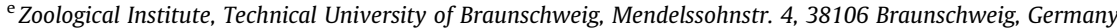 \\ ${ }^{\mathrm{f}}$ Institute of Biodiversity, Animal Health E' Comparative Medicine, College of Medical, Veterinary E Life Sciences, University of Glasgow, Glasgow, UK
}

Keywords:

Zonosaurus

Tracheloptychus

Madagascar

Biogeography

Molecular systematics

\begin{abstract}
A B S T R A C T
We analyzed the phylogenetic relationships of the Malagasy plated lizards in the family Gerrhosauridae based on DNA sequence fragments of four mitochondrial and five nuclear genes. Various clades were strongly supported by the concatenated data set and also recovered by separate analyses of mtDNA and nucDNA. In particular, two clades here named the Z. rufipes group (containing Z. bemaraha, Z. brygooi, $Z$. rufipes, $Z$. subunicolor, $Z$. tsingy and an undescribed candidate species from northern Madagascar) and the Z. ornatus group (containing Z. anelanelany, Z. laticaudatus, Z. karsteni, Z. ornatus, Z. quadrilineaus, and $Z$. trilineatus) were resolved with strong support. A third clade named the $Z$. madagascariensis group contains $Z$. madagascariensis with a nested $Z$. haraldmeieri; the status of that species requires further investigation. Tentatively we also include $Z$. aeneus in this species group although its phylogenetic relationships were poorly resolved. A fourth clade with less support included Z. boettgeri and Z. maximus. The phylogenetic position of the genus Tracheloptychus remains uncertain: whereas in the species tree it was recovered as the sister group to Zonosaurus, other methods indicated that it was nested within Zonosaurus, albeit alternative topologies were rejected with only marginal statistical support.
\end{abstract}

\section{Introduction}

Madagascar has a rich biota characterized by a high degree of endemism, which extends beyond the species level and often to the level of genera or even families (Goodman and Benstead, 2003). Among the terrestrial vertebrates of the island, there are taxa whose closest evolutionary relationships are to Asian and South American species (Noonan and Chippindale, 2006; Warren et al., 2010; Samonds et al., 2012), but the majority of colonizations probably originated from ancestors rafting over the Mozambique Channel from mainland Africa (Yoder and Nowak, 2006). Such out of Africa rafting is particularly obvious in cases where the Malagasy clades are deeply nested within exclusively African groups, e.g., in frogs of the family Hyperoliidae (Vences et al., 2003; Wollenberg et al., 2007), in lamprophiid snakes (Nagy et al., 2003), or in plated lizards of the family Gerrhosauridae (Crottini et al., 2012b).

\footnotetext{
* Corresponding author. Fax: +495313918198.

E-mail address: m.vences@tu-bs.de (M. Vences).
}

Malagasy plated lizards are represented by two genera of Ger rhosauridae: Tracheloptychus, with two species inhabiting the sub arid south and south west, and Zonosaurus, with 17 species distributed across the different biomes of the island. Gerrhosauri dae is the sister group of the exclusively African girdle tailed liz ards (family Cordylidae) and both families together comprise the unranked clade Cordyliformes (Lang, 1991; Mouton and Van Wyk, 1997; Frost et al., 2001; Lamb et al., 2003; Townsend et al., 2004; Conrad, 2008). Crown group cordyliforms are restricted to sub Saharan Africa and Madagascar, though fossils related to these lizards have been recovered from Asia and Europe (Conrad, 2008). A Cretaceous era Malagasy cordyliform fossil has been discovered (Krause et al., 2003) but was tentatively attributed to the Cordyli dae and thus probably is not closely related to the island's extant gerrhosaurids.

The monophyly of the Cordyliformes (Cordylidae + Gerrhosauri dae) is not disputed (Conrad, 2008), yet the few published molec ular studies to date (Frost et al., 2001; Odierna et al., 2002; Lamb et al., 2003; Stanley et al., 2011) focus on either one or the other of the families and, hence, a comprehensive molecular assessment 
of cordyliform relationships is wanting. Karyological analyses indi cated a high uniformity of chromosomal number among cordyli forms, especially among gerrhosaurid taxa, and therefore were not informative regarding cordyliform phylogeny (Odierna et al., 2002). The first analysis of molecular phylogenetic relationships within Cordylidae was based on mitochondrial data (Frost et al. 2001). More recently Stanley et al. (2011) conducted a more exhaustive study of Cordylidae, including mitochondrial (mt)DNA and nuclear (nuc)DNA, and proposed 10 monophyletic genera in this sub Saharan family. For the Gerrhosauridae, the only morpho logical phylogenetic analysis is that of Lang (1991) who found the Malagasy genera (Tracheloptychus and Zonosaurus) to be monophy letic and sister to a clade of African genera (Angolosaurus, Cordylo saurus, Gerrhosaurus, Tetradactylus). Lamb et al. (2003) included representatives of all gerrhosaurid genera in their analysis of four mitochondrial genes and synonymized Angolosaurus with Gerrho saurus. They also found moderate support for the reciprocal mono phyly of African and Malagasy taxa.

For Malagasy gerrhosaurids, based primarily on external mor phological data Lang (1990) proposed that Tracheloptychus was sister to a monophyletic Zonosaurus. Within Zonosaurus, a basal trichotomy separated clades containing (i) $Z$. maximus, $Z$. ornatus and $Z$. boettgeri, (ii) $Z$. trilineatus and $Z$. quadrilineatus, and (iii) all remaining species. In the latter clade (iii), two exemplars of $Z$. karsteni and Z. laticaudatus split off in a further trichotomy, fol lowed by a clade containing $Z$. madagascariensis and $Z$. haraldmei eri, which was sister to a clade containing all species with three supralabial scales anterior to the subocular (at that time, $Z$. aen eus, $Z$. rufipes and the yet unnamed $Z$. brygooi). Taxonomic revi sions have since demonstrated the existence of additional species in Zonosaurus (e.g., Vences et al., 1996; Raselimanana et al., 2000, 2006) and molecular studies (Odierna et al., 2002; Yo der et al., 2005; Raselimanana et al., 2009) have challenged the relationships within Malagasy plated lizards, despite only low support for most of the basal relationships within this group of lizards.

In order to provide a better resolved phylogenetic hypothesis for Malagasy Gerrhosauridae, we assembled a data set of four mitochondrial and five nuclear loci ( $4.7 \mathrm{k} \mathrm{bp}$ total) for most species in this group. Our results confirm Malagasy gerrhosaurids (Zono saurus + Tracheloptychus) and the genus Tracheloptychus as mono phyletic groups. The monophyly of Zonosaurus relative to Tracheloptychus remains ambiguous, but we identify several highly supported main clades within the genus Zonosaurus.

\section{Materials and methods}

\subsection{Sampling}

Samples and specimens were obtained during fieldwork in Madagascar from 2000 to 2010 (see Supplementary materials for a Table of all voucher specimens and a map of collecting localities, Fig. S1). Lizards were collected by diurnal opportunistic searches and pitfall trapping, euthanised with an overdose of MS222 or chlorobutanol, fixed in formalin and preserved in $70 \%$ ethanol. Tis sue samples from femur muscle or tail were taken before fixation and preserved separately in 95 99\% ethanol or EDTA. Specimens were deposited in the collections of the Universite d'Antananarivo, Département de Biologie Animale (UADBA), the Zoological Mu seum Amsterdam (ZMA), and the Zoologische Staatssammlung München (ZSM). In some cases, tissue samples were taken from autotomized tails and the specimens released after unambiguous identification by morphology. Additional acronyms used: ZCMV, FGZC, MVDNA, FG/MV, field numbers of M. Vences and F. Glaw;
APR, field numbers of A.P. Raselimanana, and AM, a field number of M. Anjeriniaina.

\subsection{DNA sequencing}

DNA was extracted from alcohol and EDTA preserved muscle tissue using a Dneasy Blood and Tissue Kit (Qiagen) following the manufacturer's protocol. Fragments from the following four mtDNA genes were amplified: 12S rRNA (12S), 16S rRNA (16S), cytochrome $b$ (COB) and NADH dehydrogenase subunit 1 (ND1). Fragments of the following five nuclear genes were also amplified: brain derived neurotrophic factor (BDNF), recombination activat ing gene (RAG2), phosducin (PDC), oocyte maturation factor (CMOS) and neurotrophin 3 (NT3). PCR reactions contained $0.5 \mu \mathrm{l}$ of each $10 \mu \mathrm{M}$ primer, $0.8 \mu \mathrm{l}$ of $10 \mathrm{mM}$ dNTPs, $0.4 \mu \mathrm{l}$ Taq polymer ase (Genaxxon), $1.0 \mu \mathrm{l}$ 10X PCR buffer and $1 \mu \mathrm{l}$ of DNA. Amplifica tion followed standard cycling protocols. Primer sequences and detailed PCR conditions can be found in Supplementary materialsTable S2.

PCR products were cleaned with a SAP/CIAP enzyme protocol. The product was then cycle sequenced in both directions using the same primers as in PCR amplification and electrophoresed on an ABI 3130xl after ethanol precipitation.

Forward and reverse sequences were assembled with Sequen cher v 4.2.2. Multiple sequence alignment for each gene separately was conducted in ClustalX (Thompson et al., 1997) using default settings. Reading frames for coding genes were inferred in Mac Clade v. 4.07 (Maddison and Maddison, 2003).

All newly determined DNA sequences were submitted to Gen bank (Accession Numbers KC515098 KC515339, Table S1).

\subsection{Phylogenetic analysis}

The model of molecular evolution was inferred per gene, per type of gene (i.e. coding/non coding, nuclear/mtDNA), and per codon po sition (each separately and first and second positions combined) in MrModeltest v 2.3 (Nylander, 2002) and the best model chosen by AIC (Supplementary materialsTable S3). Hypervariable sites in the ND1, 12S and 16S rRNA genes prone to multiple substitutions and gaps in $12 \mathrm{~S}$ and $16 \mathrm{~S}$ rRNA were excluded from the analysis after run ning GBLOCKS using default parameters (Castresana, 2000).

Bayesian phylogenetic analyses (Bayesian Inference, BI) of par titioned data sets were executed in MrBayes v. 3.1.2 (Ronquist and Huelsenbeck, 2003). The combined data set (all mtDNA and nucle ar genes) was analyzed with three alternative partition strategies: 5 partitions (non coding mtDNA, mtDNA 1st and 2nd position, mtDNA 3rd position, nuclear 1st and 2nd position, nuclear 3rd po sition), nine partitions (each gene separately), or 15 partitions (mtDNA non coding, each coding gene separately with 1 st and 2nd position combined, 3rd position coding separately). Statefreq, revmat, shape, pinvar and tratio were unlinked across partitions. Branch lengths prior was set to Unconstrained: Exponential (100), which had been found to improve chain mixing in prelimin ary runs (Marshall, 2010). The temp parameter was set to 0.025 , 0.04 or 0.05 , after being decreased stepwise as needed to improve mixing. Four simultaneous chains were run for 10 million genera tions sampled every 500 generations. The first 5000 or 6000 sam ples were discarded as burn in after assessing MCMC convergence. Convergence was assumed when the chain swap information for both runs was between 0.4 and 0.8 , the average standard deviation of split frequencies was minimized, the harmonic means for run 1 and 2 at stationarity were almost identical $( \pm<0.001 \%)$, and the PSRF value was $\sim 1.001$

Harmonic mean likelihood values from different partition strategies were compared using the Bayes Factor $[2 *$ (null hypothesis alternative hypothesis)] in order to determine the 
partition model strategy that showed the highest improvement relative to all other models (Brandley et al., 2005). This was se lected as the final and most appropriate phylogenetic analysis.

Additional phylogenetic analyses were carried out under the Maximum Likelihood (ML) and Maximum Parsimony (MP) opti mality criteria. For ML the data was partitioned into non coding mtDNA and codon positions for each coding mitochondrial and nu clear gene. The analysis was run on a complete, a mitochondrial and a nuclear dataset in RaxML 7.2.8 (Stamatakis, 2006). A boot strap search with 1000 replicates using a GTRCAT model was per formed followed by ML search using GTRGAMMA. Support values were drawn on the best scoring ML tree.

For MP, bootstrapping with 2000 replicates was carried out in PAUP $^{*}$ (Swofford, 2002) using TBR branch swapping and with ten random addition sequence replicates for each bootstrap replicate.

Alternative tree topologies were compared using Shimodaira Hasegawa tests (SH tests) (Shimodaira and Hasegawa, 1999) as implemented in PAUP*, and Approximately Unbiased tests (AU tests) as implemented in Consel (Shimodaira and Hasegawa, 2001; Shimodaira, 2002) (Supplementary materialsTable S4).

A species tree phylogeny was calculated in ${ }^{*}$ BEAST 1.7.4 (Heled and Drummond, 2010) using 15 partitions run for 200 million gen erations with sampling every 40,000 generations. Sequences were grouped according to current taxonomy, with $Z$. sp. 1 from Daraina added as additional terminal taxon. The tree was calculated under the uncorrelated relaxed, lognormal clock option with fixed means and the Yule tree prior. The run was repeated four times and con vergence was assessed using Tracer v 1.5 (Rambaut and Drum mond, 2007). Such coalescent based approaches to reconstruct species phylogenies give reliable results compared to other meth ods, as they account better for incongruence between gene trees (Heled and Drummond, 2010).

Additional analyses including girdle tailed lizards and plated lizards from Africa and Madagascar were performed using previ ously published $12 \mathrm{~S}$ and $16 \mathrm{~S}$ rRNA gene sequences available on GenBank (Supplementary materialsTable S5) combined with our rRNA data generated for this study. Sequences of the lizard species Androngo trivittatus and Plestiodon fasciatus (family Scincidae) were used as outgroups. The data set was partitioned by gene and the best fitting model of evolution was inferred to be GTR + G. Bayesian analyses were run in MrBayes v. 3.1.2 using the same parameters mentioned above, except that 15 million generations were per formed and burn in was set to 7500 samples. MP and ML analyses were run as described above.

\section{Results and discussion}

\subsection{Cordyliform family phylogenetic relationships}

All plated lizards (Gerrhosauridae) were recovered as sister to a monophyletic African Cordylidae (Platysaurinae and Cordylinae). Our analysis recovered, with strong support, the split within Cord ylidae between the Platysaurus group and all other cordylids re ported by Stanley et al. (2011). Our BI, MP and ML phylogenetic analyses found all Malagasy plated lizards (Zonosaurinae; genera Zonosaurus and Tracheloptychus) to be monophyletic and sister to the African plated lizards (Gerrhosaurinae); this relationship is ro bustly supported in all analyses (Fig. S2). The Zonosaurinae are notable for the short internal branch lengths relative to the other three subfamilies of cordyliforms, especially compared to the sister group Gerrhosaurinae and its long internal branches. We suggest this may represent an initial burst of diversification in Malagasy ger rhosaurids after their colonization of the island, though this requires further testing and is beyond the scope of the current paper. The phylogenetic relationships within the well supported Zonosaurinae are the focus of our current study.

\subsection{Zonosaurinae phylogenetic relationships}

The combined dataset of mitochondrial and nuclear loci con sisted of 4708 nucleotides. A total of 3989 nucleotides were re tained for analysis after conservatively excluding all variable parts of the $12 \mathrm{~S}$ and $16 \mathrm{~S}$ loci that required gaps for alignment, and excluding terminal portions of each partition due to the prev alence of missing data. In the full data set, 3031 characters were constant and 615 were parsimony informative. The final mtDNA data set had 1852 characters of which 1167 were constant and 521 were parsimony informative. The nucDNA data set had 2059 characters of which 1864 were constant and 94 were parsimony informative.

The phylogenetic tree (BI) inferred using the combined data set contained many well supported nodes (Bayesian posterior proba bilities (PP) of 1.0) (Fig. 1). The species tree analysis (Fig. 2) re sulted in a topology largely congruent with the BI tree of the concatenated sequences, with a remarkable although only weakly supported difference (i.e., the placement of Zonosaurus and Trachel optychus as reciprocally monophyletic; see below). However, be cause effective sample size (ESS) values of some parameters in the ${ }^{*}$ BEAST analysis remained low $(<200)$ despite repeating the analysis four times, this result needs to be seen with caution. As expected, separate analyses of mtDNA and nucDNA alone resulted in less robustly supported topologies and some basal nodes were not congruent among analyses (Fig. 1). Nonetheless, several taxo nomic relationships were resolved by both data sets.

In particular, our analysis strongly supports the monophyly of the genus Tracheloptychus, with T. madagascariensis and T. petersi, and four major clades within Zonosaurus that we here define as species groups (Fig. 1). The following three species groups were strongly supported and recovered by all analyses: (i) the $\boldsymbol{Z}$. madagascariensis group that contains $Z$. haraldmeieri clustering within a paraphyletic $Z$. madagascariensis, (ii) the $Z$. rufipes group contain ing Z. bemaraha, Z. brygooi, Z. rufipes, Z. subunicolor, Z. tsingy, and an undescribed candidate species from northern Madagascar (Z. sp. 1 ), (iii) and the $Z$. ornatus group containing Z.anelanelany, Z. laticaud atus, Z. karsteni, Z. ornatus, $Z$. quadrilineatus, and Z. trilineatus. Fur thermore the combined analyses also recovered (iv) a $Z$. boettgeri group, with $Z$. maximus and $Z$. boettgeri but with less support, and without support in the MP analysis, presumably due to fewer data available (PP: ${ }^{*}$ BEAST $=1.0$, MrBayes $=0.72 ; \mathrm{ML}=63$ ).

Most of these findings are consistent with the phylogenetic analyses of Raselimanana et al. (2009), but there are some substan tial improvements to the resolution of relationships, especially for some of the deeper evolutionary nodes (Fig. 2). Tracheloptychus and our Zonosaurus clades (i) and (ii) were also supported in the tree of Raselimanana et al. (2009), whereas the $Z$. ornatus group and the $Z$. boettgeri group were not recovered in their analyses, likely due at least in part to the absence of $Z$. maximus and the reduced phy logenetic information of their smaller data set.

\subsection{Relationships of Tracheloptychus}

As in previous studies (e.g., Lang, 1991; Raselimanana et al., 2009), the monophyly of the genus Tracheloptychus is strongly sup ported by our analysis. This group consists of the two Malagasy gerrhosaurid species, T. madagascariensis and T. petersi, possessing keeled dorsal scales (in contrast Zonosaurus have smooth scales), and keeled scales underneath digits (rounded in Zonosaurus). These two species inhabit the subarid south and south west of Madagas car. Morphological studies (e.g., Lang, 1990, 1991) have so far not reported any clear synapomorphy shared by all Zonosaurus to the exclusion of Tracheloptychus, despite the obviously different gen eral appearance of these two groups of lizards. The molecular data are ambiguous as well. Tracheloptychus is consistently placed 


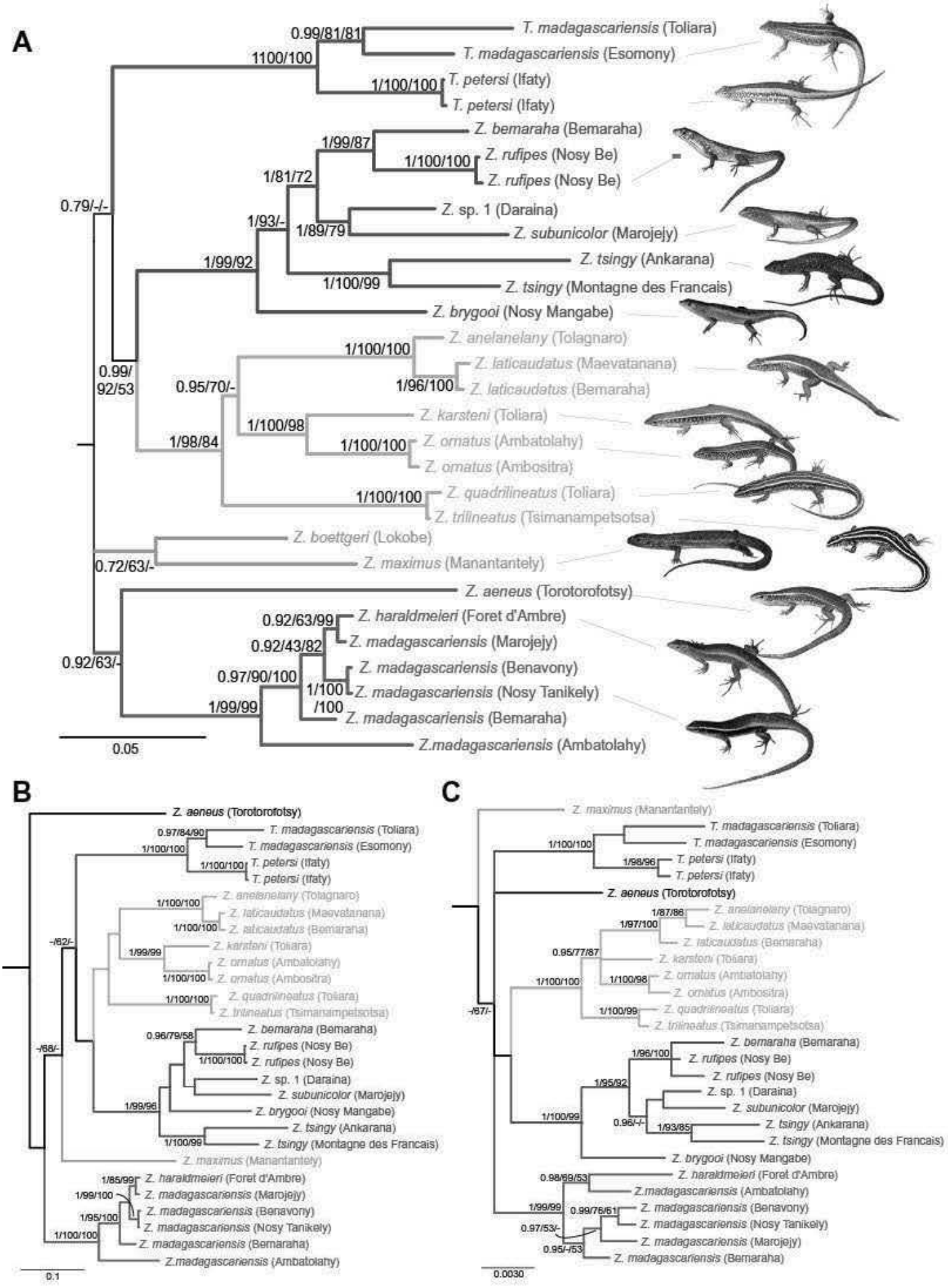

Fig. 1. Phylogenetic trees of species of Malagasy gerrhosaurids (Zonosaurus and Tracheloptychus) based on an analysis of DNA sequences of mitochondrial and/or nuclear genes. The trees are 50\%-majority rule consensus trees from a Bayesian analysis of (A) the whole set of concatenated genes, (B) the mitochondrial gene fragments only (12S, $16 S$, ND1, COB), and (C) the nuclear gene fragments only (BDNF, PDC, RAG2, CMOS, NT3). Note that Z, boettgeri was excluded from calculations based on only either mtDNA or nucDNA due to limited data. Support values are Bayesian posterior probabilities, and bootstrap proportions from ML and MP analyses respectively. Different colors mark species groups congruently suggested by the mtDNA and the nucDNA analysis, as discussed in the text (except the conflicting position of $Z$. aeneus in the $Z$ madagascariensis group). The tree was rooted using an African gerrhosaurid (Gerrhosaurus cf. nigrolineatus) as the outgroup. (For interpretation of the references to color in this figure legend, the reader is referred to the web version of this article.)

within Zonosaurus in the concatenated analyses, but mitochondrial and nuclear data do not resolve the relationships among the main clades within Malagasy plated lizards, including Tracheloptychus (indicated by low support for the topology of deep nodes, Fig. 1).
The species tree analysis placed Tracheloptychus sister to a mono phyletic Zonosaurus (Fig. 2) with low support $(\mathrm{PP}=0.62)$ and this position was not significantly excluded by $\mathrm{AU}$ (near significant, $p=0.054$ ) and SH tests (Supplementary materialsTable S4 and 


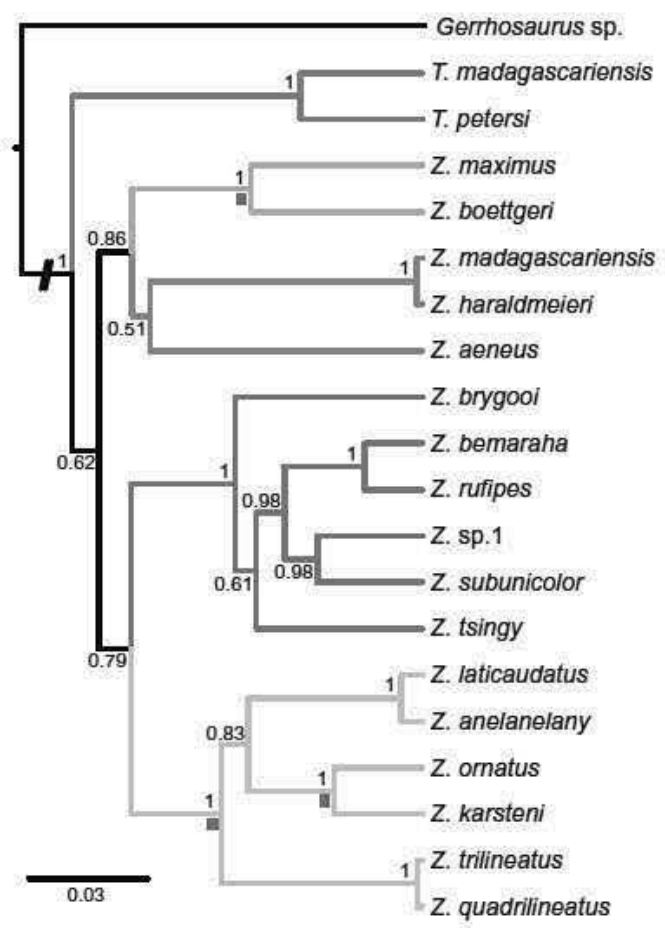

Fig. 2. Species tree phylogeny of Malagasy gerrhosaurids as obtained by the "BEAST species tree reconstruction method. Colors refer to main clades (as in Fig. 1). Bayesian posterior probabilities are indicated on each node. Nodes with high support values ( $\mathrm{PP}>0.95$ ) that were not resolved in previous studies are indicated with a gray square. Four independent runs of the species tree analysis resulted in identical topologies and effective sample size (ESS) values $>200$ in the majority of parameters, including the likelihood values. (For interpretation of the references to color in this figure legend, the reader is referred to the web version of this article.)

Fig. S3). Although it is possible that Tracheloptychus should be con sidered a synonym of Zonosaurus, more data are needed before for malizing this conclusion taxonomically.

\subsection{Relationships within Zonosaurus species groups}

The $Z$. boettgeri group as defined herein consists of $Z$. boettgeri and $Z$ maximus in our present phylogeny. The clade was well supported in the species tree analysis (PP - 1.0) (Fig. 2), and less well supported in the combined analysis ( $P P=0.72, M L=63$ ) (Fig. 1). A third species, Z. maramaintso, could not be included in our analysis because we had no sample available and there are no sequence data for it available in public databases, but is pre sumably closely related to $Z$. boettgeri (Raselimanana et al., 2006). Lang (1990) also placed $Z$. boettgeri and $Z$. maximus together in a clade that additionally contained $Z$. ornatus. He based this grouping on obliquely keeled plantar scales found in $Z$ ornatus and $Z$ maximus (and $Z$. karsteni), and fused prefrontal scales in $Z$ boettgeri and $Z$. maximus (plus several other species for which convergent evolution of this character state was assumed). Hence, he did not identify any synapomorphy exclusively shared by $Z$ boettgeri and $Z$. maximus. Statistical tests of the molecular data (Fig. S3) rejected $\mathrm{Z}$ ornatus belonging to this clade, indicating mor phological convergence of the keeled plantar scales in $Z$. maximus on one hand and the $Z$. ornatus/karsteni clade on the other hand. $Z$ boettgeri and probably $Z$. maramaintso are specialized arboreal lizards with a very long tail while $Z$. maximus is a large sized semi aquatic lizard with a laterally compressed tail. One possible synapomorphy of these taxa is their comparatively low number of dorsal scale rows (14 16 in $Z$. boettgeri and $Z$ maramaintso, and 1821 in $Z$ maximus, vs. reaching more than 21 in all other species; Brygoo and Böhme 1985).

Our data are in agreement with previous molecular studies (Yoder et al., 2005; Raselimanana et al., 2009) in suggesting that $Z$ haraldmeieri, a species from the extreme north of Madagascar, is phylogenetically deeply nested within the widespread Z madagascariensis, making $Z$ madagascariensis paraphyletic (Fig. 1). We suggest naming this clade the $Z$. madagascariensis group (into which we also tentatively place $Z$, aeneus; see discus sion on that species below). The paraphyly of $Z$. madagascariensis is supported by both mitochondrial and nuclear markers, though the relationships within this group are not consistent across mark ers; nucDNA analyses placed $Z$. haraldmeieri sister to the southern most Z. madagascariensis included (from Ambatolahy) that in mtDNA was most divergent from all other individuals. Given that morphological differences between these two species are re stricted to color (uniform greenish in Z. haraldmeieri vs. distinct dorsolateral stripes in Z madagascariensis; see Lang, 1990), the spe cies status of $Z$ haraldmeieri is doubtful (Raselimanana et al., 2009). The differentiation of $Z$. haraldmeieri in at least some nuclear genes (see also Raselimanana et al., 2009) suggests that the status of this taxon also requires more detailed population genetic work. The occurrence of a green colored isolated population ( $Z$ haraldmeieri) in northernmost Madagascar, nested within the widespread brown colored Z. madagascariensis, bears a conspicuous similarity to the pattern encountered in the frogs Mantella viridis and $M$. ebe naui; in these, populations with these different color patterns in northern Madagascar are divided by a barrier to gene flow despite widespread haplotype sharing, and this barrier might be related to bioclimatic differences (Crottini et al., 2012a).

The phylogenetic position of $\mathrm{Z}$ aeneus remains unsolved. It forms the sister group of the $Z$ madagascariensis/haraldmeieri clade in the species tree and the concatenated analyses, but with little congruence across genes and rather low support (Figs. 1 and 2). We here include this species tentatively in the $Z$. madagascariensis group considering also its striking similarity to $Z$. madagascariensis in color pattem (Glaw and Vences, 2007), but we are aware that this classification might require revision once that new data be come available.

The $Z$ rufipes group as defined herein contains small sized, for est dwelling Zonosaurus species that are characterized by the pres ence of three rather than four supralabials anterior to the subocular (Vences et al., 1996). MtDNA and nucDNA supported close relationships between $Z$. bemaraha and $Z$. rufipes, and of $Z$ subunicolor with $Z$. sp. 1 from Daraina. Either $Z$. tsingy (in the mtDNA data set) or Z brygooi (in the nucDNA and complete data set) resolved as sister to those species in this clade (Fig. 1).

Besides species of the $Z$. rufipes group and both species of the genus Tracheloptychus, two other species of Malagasy plated lizards are characterized by three supralabials anterior to the subocular and smaller body size: $Z$ aeneus and $Z$. anelanelany. Phylogenetic arrangements that place all taxa with this morphological character in a monophyletic group or otherwise reduce the homoplasy for this character could in most cases be rejected by our analyses as detailed in the following: None of our phylogenetic analyses place $\mathrm{Z}$ aeneus or $\mathrm{Z}$. anelanelany in the $\mathrm{Z}$. rufipes group, nor are these two species recovered as sister taxa (Figs. 1 and 2). Alternative topologies in which these two species are constrained hierarchi cally as sister to the Z rufipes group (per Lang, 1991) or sister to the rest of the Malagasy gerrhosaurids are significantly rejected by $\mathrm{SH}$ and $\mathrm{AU}$ tests $(p<0.001$; alternative trees 2 and 3 in Supple mentary materials, Fig. S3 and Table S4) and none of them is sup ported by the species tree (Fig. 2). Another alternative topology in which only $Z$. aeneus is sister to the rest of the $Z$ rufipes group is only marginally rejected by the AU test $(p=0.056)$ and is not re jected by the $\mathrm{SH}$ test (Table S4). Furthermore, the alternative 
topologies of Tracheloptychus as the sister group to all Zonosaurus, or similarly, an alternative with $Z$. aeneus (but not $Z$. anelanelany) sister to the $Z$. rufipes group and Tracheloptychus moved to become the sister group of all Zonosaurus are not rejected by any of the tests (trees 5 and 6 inSupplementary materials).

In previous assessments (e.g., Lang, 1990; Vences et al., 1996; Vences et al., 1999), Z. aeneus was included with Z. brygooi, $Z$. rufipes, and $Z$. subunicolor in a clade named the " $Z$. aeneus group". Yet our current analyses clearly place $Z$. aeneus phylogenetically distant from these other species (Figs. 1 and 2).Z. aeneus inhabits rather open areas within forest or at forest edges, while species of the $Z$. rufipes group are typically found in sunlit spots within rather dense forest (Vences et al., 1996; Raselimanana et al., 2000; Glaw and Vences, 2007). This difference in the species' ecol ogy is thus in agreement with the lack of close relationships among $Z$. aeneus and the $Z$. rufipes group. Interestingly, $Z$. sp. 1 from Daraina, which unambiguously resolves sister to $Z$. subunicolor, bears a strong morphological similarity to $Z$. aeneus while occurring in more dense parts of the forest (A.P. Raselimanana, unpublished data), highlighting that morphology alone is a poor indicator of phylogenetic relatedness in these lizards.

Homoplasy in the evolution of body size and supralabial config uration in Malagasy plated lizards is furthermore suggested by the strongly supported position of $Z$. anelanelany sister to the included samples of $Z$. laticaudatus. The data of Raselimanana et al. (2009) indicated that $Z$. anelanelany is phylogenetically nested within Z. laticaudatus, being more closely related to those Z. laticaudatus populations occurring in the south of Madagascar. Single gene analysis of our data revealed that, besides mtDNA, three nuclear genes (BDNF, CMOS, RAG2) support close relationships of $Z$. ane lanelany to $Z$. laticaudatus (not shown). A further gene (NT3) had too little variability to reveal any clear grouping, and no $Z$. anelane lany sequence was obtained for PDC. The congruent placement of $Z$. anelanelany with $Z$. laticaudatus by four independent markers (combined mtDNA, BDNF, CMOS, RAG2) unambiguously suggests close phylogenetic relationships among these taxa rather than a confounding pattern of mtDNA introgression. In our analysis, only a single sample of $Z$. anelanelany was included, but the same pat tern was found based on multiple specimens (but fewer markers) studied by Raselimanana et al. (2009) and sample confusion is therefore unlikely. $Z$. laticaudatus and $Z$. anelanelany occur sympat rically in the extreme south east of Madagascar, but use different habitats (rocky substrate and open areas vs. almost closed canopy humid forest) and maintain their clear morphological distinctness. Our analysis did not include samples of southern $Z$. laticaudatus, which share CMOS haplotypes with $Z$. anelanelany (Raselimanana et al., 2009). More fieldwork in the contact zones of these two spe cies is needed to understand their population genetic, morpholog ical and ecological differentiation.

The $\boldsymbol{Z}$. ornatus group as defined herein mostly contains species specializing in arid and subarid habitats in western and southern Madagascar plus $Z$. ornatus, which inhabits montane ericoid and thicket vegetation as well as rainforest edges in the middle and southern central east, and $Z$. anelanelany, which occurs in south eastern humid environments. The molecular study by Raselimanana et al., (2009) did not detect this clade; however, all our analyses strongly support its monophyly (Figs. 1 and 2). Zonosaurus laticaudatus is the most generalist species in this group with regard to habitat (wet or arid, gallery forest, dry forest, rocky or limestone), while $Z$. ornatus occurs over the widest elevational range (from near sea level in Manombo National Park, to above $2000 \mathrm{~m}$ a.s.l. in the Andringitra and Ankaratra Massifs). Both mtDNA and nucDNA analyses agree in placing the two closely related and morphologically similar species, Z. quadrilineatus and Z. trilineatus, as sister to the rest of this clade. Furthermore, $Z$. karsten $i$ is resolved as sister species of $Z$. ornatus and as mentioned above, $Z$. anelanelany is sister to Z. laticaudatus. Previous analyses (Lang, 1990; Raselima nana et al., 2009) unambiguously placed $Z$. quadrilineatus and $Z$. trilineatus together in a clade. A further study based on $16 \mathrm{~S}$ sequences only and with limited taxon sampling obtained a clade containing Z. karsteni, Z. ornatus and Z. trilineatus (Odierna et al., 2002). Therefore multiple lines of evidence support this biological grouping.

\section{Conclusions}

While the broad level relationships of the cordyliforms are fairly well established (Conrad, 2008), internal relationships within the Gerrhosauridae have remained conflicted or less well resolved (e.g. Lang, 1991; Odierna et al., 2002; Lamb et al., 2003; Raselima nana et al., 2009). Our present study has contributed information from mitochondrial and nuclear molecular markers and sought to clarify some of the relationships with Zonosaurinae in particular. Our analysis strongly supports five internal groups, of which two have not been detected previously: Tracheloptychus, the $Z$. rufipes group, the $Z$. madagascariensis group, and the newly identified $Z$. ornatus and $Z$. boettgeri groups. Questions remain as to the valid ity of the genus Tracheloptychus, which might render Zonosaurus paraphyletic. However, the species tree supported Tracheloptychus being sister to Zonosaurus (Fig. 2) which differs from our combined tree including all specimens (Fig. 1) and suggests that more exten sive molecular data will be required to clarify the relationships. Some of the basal nodes within the Malagasy Gerrhosauridae thus remain unresolved and may require even more data to increase resolution. We hypothesize that there has been a fast initial radia tion of the Malagasy gerrhosaurids that makes it difficult to resolve the deeper nodes of this phylogenetic group.

\section{Acknowledgments}

We are grateful to Franco Andreone, Parfait Bora, Frank Glaw, Roger Daniel Randrianiaina, and David R. Vieites for assistance and companionship in the field during the collection of crucial samples, to Susanne Hauswaldt, Gabi Keunecke, Meike Konder mann, and Eva Saxinger for their help in the lab, to Aurelien Miral les who kindly made photographs available, and to two anonymous reviewers who considerably helped improving a previ ous version of the manuscript. We also thank the Leonardo da Vinci programme and Barbara Helm for support. Fieldwork of M.V. in Madagascar was made possible by collaboration agreements of the author's institutions with the Université d'Antananarivo (Département de Biologie Animale) and the Madagascar National Parks (formerly Association Nationale pour la Gestion des Aires Protegées), and was supported by the Volkswagen Foundation and the Deutsche Forschungsgemeinschaft. We thank the Mala gasy authorities for issuing collection and export permits.

\section{References}

Brandley, M., Schmitz, A., Reeder, T., 2005. Partitioned Bayesian analyses, partition choice, and the phylogenetic relationships of scincid lizards. Syst. Biol. 54, 373390.

Brygoo, E.R., Böhme, W., 1985. Un Zonosaurus nouveau de la région d’Antseranana (Diégo Suarez, Madagascar) (Reptilia: Cordylidae). Revue Française d'Aquariologie et Herpétologie 12, 31-32.

Castresana, J., 2000. Selection of conserved blocks from multiple alignments for their use in phylogenetic analysis. Mol. Biol. Evol. 17, 540-552. 
Conrad, J.L., 2008. Phylogeny and systematics of Squamata (Reptilia) based on morphology. Bull. Am. Mus. Nat. Hist. 310, 1-182.

Crottini, A., Brown, J.L., Mercurio, V., Glaw, F., Vences, M., Andreone, F., 2012a. Phylogeography of the poison frog Mantella viridis (Amphibia: Mantellidae) reveals chromatic and genetic differentiation across ecotones in northern Madagascar. J. Zool. Syst. Evol. Res. 50, 305-314.

Crottini, A., Madsen, O., Poux, C., Strauß, A., Vieites, D.R., Vences, M., 2012b. Vertebrate time-tree elucidates the biogeographic pattern of a major biotic change around the K-T boundary in Madagascar. Proc. Natl. Acad. Sci. USA 109, 5358-5363.

Frost, D., Janies, D., Mouton, P., LeF, N., Titus, T., 2001. A molecular perspective of the girdled lizards. Am. Mus. Novit. 3310, 1-10.

Glaw, F., Vences, M., 2007. A Field Guide to the Amphibians and Reptiles of Madagascar, third ed. Vences and Glaw Verlag, Köln (Cologne).

Goodman, S.M., Benstead, J.P. (Eds.), 2003. The Natural History of Madagascar. The University of Chicago Press, Chicago.

Heled, J., Drummond, A.J., 2010. Bayesian inference of species trees from multilocus data. Mol. Biol. Evol. 27, 570-580.

Krause, D.W., Evans, E.E., Gao, K.-Q., 2003. First definitive record of Mesozoic lizards from Madagascar. J. Vert. Paleontol. 23, 842-856.

Lamb, T., Meeker, A.M., Bauer, A.M., Branch, W.R., 2003. On the systematic status of the desert plated lizard (Angolosaurus skoogi): phylogenetic inference from DNA sequence analysis of the African Gerrhosauridae. Biol. J. Linn. Soc. 78, 253-261.

Lang, M., 1990. Phylogenetic analysis of the genus group TracheloptychusZonosaurus (Reptilia: Gerrhosauridae) with the hypothesis of biogeographical unit relationships in Madagascar. In: Peters, G., Hutterer, R. (Eds.), Vertebrates in the Tropics. Museum Alexander Koenig, Bonn, pp. 261-274.

Lang, M., 1991. Generic relationships within Cordyliformes (Reptilia: Squamata). Bull. Inst. Royal Sci. Nat. Belg. Biol. 61, 121-188.

Maddison, D.R., Maddison, W.P., 2003. MacClade 4: Analysis of Phylogeny and Character Evolution. Version 4.07. Sinauer Associates, Sunderland, MA, USA.

Marshall, D.C., 2010. Cryptic failure of partitioned Bayesian phylogenetic analyses: lost in the land of long trees. Syst. Biol. 59, 108-117.

Mouton, P.L.F.N., Van Wyk, J.H., 1997. Adaptive radiation in cordyliform lizards: an overview. Afr. J. Herpetol. 46, 78-88.

Nagy, Z.T., Joger, U., Wink, M., Glaw, F., Vences, M., 2003. Multiple colonization of Madagascar and Socotra by colubrid snakes: evidence from nuclear and mitochondrial gene phylogenies. Proc. R. Soc. Lond. B. 270, 2613-2621.

Noonan, B., Chippindale, P., 2006. Vicariant origin of Malagasy reptiles supports Late Cretaceous Antarctic land bridge. Am. Nat. 168, 730-741.

Nylander, J.A.A., 2002. MrModeltest v1.0b. Program Distributed by the Author. <http://www.ebc.uu.se/systzoo/staff/nylander.html>.

Odierna, G., Canapa, A., Andreone, F., Aprea, G., Barucca, M., Capriglione, T., Olmo, E., 2002. A phylogenetic analysis of Cordyliformes (Reptilia: Squamata): comparison of molecular and karyological data. Mol. Phylogenet. Evol. 23, 3742.

Rambaut, A., Drummond, A.J., 2007. Tracer v1.4. Available from <http:// beast.bio.ed.ac.uk/Tracer>

Raselimanana, A.P., Noonan, B., Karanth, K.P., Gauthier, J., Yoder, A.D. 2009. Phylogeny and evolution of Malagasy plated lizards. Mol. Phylogenet. Evol. 50, 336-344.

Raselimanana, A.P., Nussbaum, R.A., Raxworthy, C.J., 2006. Observations and redescription of Zonosaurus boettgeri Steindachner 1891 and description of a second new species of long-tailed Zonosaurus from western Madagascar. Occasional Papers Museum of Zoology, University of Michigan, vol. 739, pp. $1-16$.
Raselimanana, A.P., Raxworthy, C.J., Nussbaum, R.A., 2000. A revision of the dwarf Zonosaurus Boulenger (Reptilia: Squamata: Cordylidae) from Madagascar, including descriptions of three new species. Scientific Papers, The Natural History Museum, University of Kansas, vol. 18, pp. 1-16.

Ronquist, F., Huelsenbeck, J.P., 2003. MrBayes 3: Bayesian phylogenetic inference under mixed models. Bioinformatics 19, 1572-1574.

Samonds, K.E., Godfrey, L.R., Ali, J.R., Goodman, S.M., Vences, M., Sutherland, M.R., Irwin, M.T., Krause, D.W., 2012. Spatial and temporal arrival patterns of Madagascar's vertebrate fauna explained by distance, ocean currents, and ancestor type. Proc. Natl. Acad. Sci. USA 109, 5352-5357.

Shimodaira, H., 2002. An approximately unbiased test of phylogenetic tree selection. Syst. Biol. 51, 492-508.

Shimodaira, H., Hasegawa, M., 1999. Multiple comparisons of log-likelihoods with applications to phylogenetic inference. Mol. Biol. Evol. 16, 1114-1116.

Shimodaira, H., Hasegawa, M., 2001. CONSEL: for assessing the confidence of phylogenetic tree selection. Bioinformatics 17, 1246-1247.

Stamatakis, A., 2006. RAxML-VI-HPC: maximum likelihood-based phylogenetic analyses with thousands of taxa and mixed models. Bioinformatics 22, 26882690.

Stanley, E.L., Bauer, A.M., Jackman, T.R., Branch, W.R., Mouton, P., LeF, N., 2011. Between a rock and a hard polytomy: rapid radiation in the rupicolous gridled lizards (Squamata: Cordylidae). Mol. Phylogenet. Evol. 58, 53-70.

Swofford, D.L., 2002. PAUP*. Phylogenetic Analysis using Parsimony ("and other methods). Version 4.0b10. Sinauer Associates, Sunderland, MA.

Thompson, J.D., Gibson, T.J., Plewniak, F., Jeanmougin, F., Higgins, D.G., 1997. The CLUSTAL X windows interface: flexible strategies for multiple sequence alignment aided by quality analysis tools. Nucl. Acids Res. 25 (24), 4876-4882.

Townsend, T.M., Larson, A., Louis, E., Macey, J.R., 2004. Molecular phylogenetics of Squamata: the position of snakes, amphisbaenians, and dibamids, and the root of the squamate tree. Syst. Biol. 53, 735-757.

Vences, M., Kosuch, J., Glaw, F., Böhme, W., Veith, M., 2003. Molecular phylogeny of hyperoliid treefrogs: biogeographic origin of Malagasy and Seychellean taxa and re-analysis of familial paraphyly. J. Zool. Syst. Evol. Res. 41, 205-215.

Vences, M., Müller-Jung, J., Glaw, F., Böhme, W., 1996. Review of the Zonosaurus aeneus species group, with resurrection of Zonosaurus subunicolor (Boettger 1881) (Reptilia: Squamata: Gerrhosauridae). Senckenbergiana Biol. 76 (1/2), $47-59$.

Vences, M., Ziegler, T., Visser, S., Andreone, F., 1999. New data on the zoogeography and genital morphology of the lizards Zonosaurus brygooi Lang \& Böhme 1990 and Z. aeneus (Grandidier 1872) from Madagascar (Reptilia Squamata Gerrhosauridae). Tropical Zool. 12 (1), 145-155.

Warren, B.H., Strasberg, D., Bruggemann, J.H., Prys-Jones, R.P., Thébaud, C., 2010. Why does the biota of the Madagascar region have such a strong Asiatic flavour? Cladistics 26, 526-538.

Wollenberg, K.C., Glaw, F., Meyer, A., Vences, M., 2007. Molecular phylogeny of Malagasy reed frogs, Heterixalus, and the relative performance of bioacoustics and color-patterns for resolving their systematics. Mol. Phylogenet. Evol. 45, $14-22$.

Yoder, A.D., Nowak, M., 2006. Has vicariance or dispersal been the predominant biogeographic force in Madagascar? Only time will tell. Annu. Rev. Ecol. Evol. Syst. 37, 405-431.

Yoder, A.D., Olson, L.E., Hanley, C., Heckman, K.L., Rasoloarison, R., Russell, A.L., Ranivo, J., Soarimalala, V., Karanth, K.P., Raselimanana, A.P., Goodman, S.M., 2005. A multidimensional approach for detecting species patterns in Malagasy vertebrates. Proc. Natl. Acad. Sci. USA 102, 6587-6594. 